\title{
Features stabilization of the consumer current using electro- ferromagnetic circuits
}

\author{
G.R. Rafikova ${ }^{1}, M . R$. Ruzinazarov $^{1}$, S.K. Makhmutkhonov ${ }^{1}$ \\ ${ }^{1}$ Tashkent State Technical University named after Islam Karimov, 100095, Uzbekistan, Tashkent, University St. 2A
}

\begin{abstract}
Electromagnetic ferromagnetic oscillatory circuits having falling sections on the amplitude characteristics formed the basis of stabilizers based on the principle of summing the currents of individual branches. A distinctive feature of such stabilizers is that the current remains unchanged both when the load resistance changes, so when the input voltage varies over a wide range. This is required by devices such as electromagnets of physical instruments, electromagnetic lenses of electron microscopes, instruments for emitting nuclear paramagnetic resonance, instruments and devices of various automation schemes, measuring and computing equipment, as well as many electrotechnological processes. Therefore, current stabilization has long attracted the attention of many researchers, and this is still one of the important problems of electrical engineering. The curve shape of the proposed current stabilizer is close to a sinusoid, the power factor is very high, since the device operates in capacitive mode.
\end{abstract}

In the modern system, in the power supply circuit of various devices, electro-ferromagnetic circuits are widely used as current and voltage stabilizers, phase and frequency converters, contactless switching devices, since devices created on the basis of electroferromagnetic oscillatory circuits have high operational reliability and resistance to mechanical overloads, fairly high efficiency, $\cos \varphi$, manufacturing limit from several watts to tens of $\mathrm{kW}$, low cost.

Interest in electromagnetic circuits has also increased with the rapid development of semiconductor technology, because in these circuits peculiar phenomena arise that are not fundamentally observed in linear systems, in particular, autoparametric oscillations are possible at the source frequency and at other frequencies (higher, subharmonic and Raman oscillations).

In this case, currents and voltages on individual elements of the circuit abruptly change, or stable negative sections can be observed on the amplitude and phase characteristics of the circuit $[1,2,5]$.

The practical use of phenomena in electromagnetic circuits, where single-circuit, double-circuit circuits are considered, opens up new possibilities for their application in the creation of electrical devices.

In electromagnetic vibrational circuits (EFVCC), a coil with a ferrimagnetic core introduces nonlinearity, which leads to the occurrence of phenomena not observed in linear circuits. The simplest EFVCC, consisting of a nonlinear inductance and a linear capacitance connected in parallel, has an "S" -shaped current-voltage characteristic. By sequentially switching on a parallel line of linear inductance, it is possible to expand the zone of the volt-ampere characteristic of the negative section $[3,7]$. Based on the compensation of the incident section, a new principle of current stabilization is proposed by summing the current-voltage characteristics of individual circuit elements, ensuring a minimum specific gravity of circuit elements and an almost sinusoidal shape of the stabilized current curve.

The use of the incident section on the amplitude characteristics as sensitive organs in the control circuits of amplifying and thyristor devices can significantly improve the overall dimensions of stabilizing devices, while retaining the advantages of controlled semiconductor, ferrimagnetic elements and ferroresonant circuits [7-10].

To analyze the steady state of a three-element oscillatory circuit, we use the circuit equivalent circuit shown in Fig. 1. where the following assumptions are made:

Losses in EFVCC are taken into account by the active conductivity $g$ connected in parallel with the nonlinear element. The $\mathrm{g}$ value is considered constant over the entire range of input voltage variation.

1. Active resistances of linear elements are taken into account.

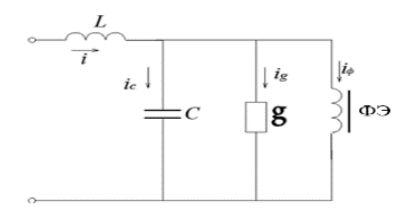

Fig. 1. EFVCC equivalent circuit 
$L C W \frac{d^{3} \Phi}{d t^{3}}+L g W \frac{d^{2} \Phi}{d t^{2}}+W \frac{d \Phi}{d t}+\frac{L K}{W} \frac{d \Phi^{n}}{d t}=u$

here is the u-applied voltage;

$\mathrm{W}$ - the number of turns of the winding of the ferromagnetic element;

$\Phi$ - main magnetic flux in the core of the ferromagnetic element;

$$
\phi=\Phi_{\mathrm{m}} \sin \omega \mathrm{t}
$$

$\mathrm{n}$ - degree of approximating function. For electrical steel, it is advisable to take $n=7$ or $n=9$.

We solve the problem for the general case:

After reducing to relative units and introducing some notation from (1.1) we get:

$$
y=\frac{d^{3} x}{d \tau^{3}}+\delta \frac{d^{2} x}{d \tau^{2}}+\frac{1}{A} \frac{d x^{n}}{d \tau}+\beta \frac{d x}{d \tau},
$$

Here

$$
\begin{gathered}
y=\frac{u}{u_{\tilde{\sigma}}} ; \quad x=\frac{\Phi}{\Phi_{\sigma}} ; \quad \sigma=\frac{g}{\omega c} ; \quad \beta=\frac{1}{\omega^{2} L C} ; \quad \tau=\omega t \quad U_{\sigma}=\omega^{3} W L C \Phi_{\bar{\sigma}} \\
\Phi_{\sigma}=\sqrt[n-1]{\frac{W^{2} \omega^{2} C}{A K}}
\end{gathered}
$$

The chain equation (2), taking into account dimensionless coefficients, has the following form:

$$
\begin{gathered}
Y^{2}{ }_{m}=\delta^{2} X^{2}{ }_{m}+X^{2}{ }_{m}\left(X^{n-1}{ }_{m}-1+\beta\right)^{2} \\
\operatorname{tg} \psi=\frac{\delta}{X_{m}^{n-1}-1+\beta}
\end{gathered}
$$

We find the current-voltage characteristic using the expression of current in a dimensionless form $Z_{m}=\frac{I_{m}}{I_{\delta}}$

$$
Z_{m}=X_{m} \sqrt{\left(X_{m}^{n-1}-1\right)^{2}+\delta^{2}}, \quad \operatorname{tg} \psi=\frac{\delta}{X_{m}^{n-1}-1}
$$

In fig. 2 - 5 show a series of characteristics for various circuit parameters and the influence of the source frequency.

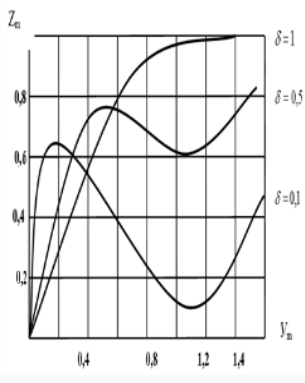

Fig. 2. Dependencies $Z_{m}=f\left(Y_{m}\right)$

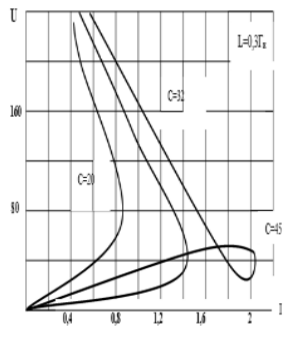

Fig. 4. Dependencies $U=f(I)$ for various $\mathrm{C}$

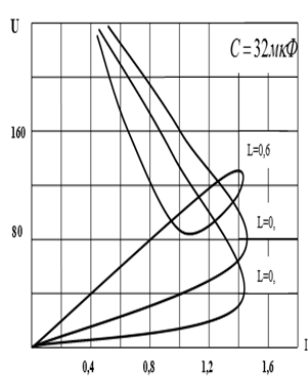

Fig. 5. Dependences $U=f(I)$ for various $L$
The following conclusions can be drawn from an analysis of the results:

1 All parameters of the circuit and the frequency of the power source significantly affect the width of the zone of the falling part of the current-voltage characteristics of the EFVCC;

2. The width of the falling part of the characteristic substantially depends on the value of the capacitance, with an increase in this parameter the resonance point shifts to the region of high stresses and this leads to the expansion of the zone of the negative part of the characteristic;

3. By varying the value of the parameter $\mathrm{L}$, it is possible to change the slope of the falling part of the amplitude characteristic.

Here is an analysis of the current stabilizer (Fig. 6.) based on the principle of summing the currents of two mutually parallel branches, the stabilization principle makes it possible to increase the output power, improve the stabilization coefficient, expand the allowable range of input voltage variation and reduce the proportion of higher harmonic components in the load current.

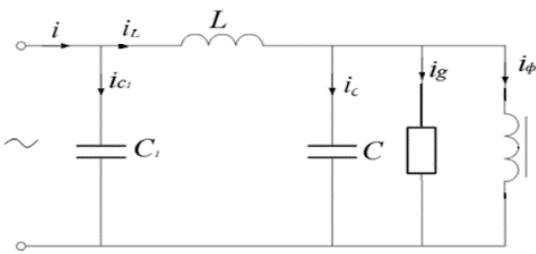

Fig. 6. Equivalent circuit CS

$$
i=i_{c 1}+i_{c}+i_{p}+i_{g}
$$

Where

$i_{g}=g W \frac{d \Phi}{d t} ; \quad i_{C}=C W \frac{d^{2} \Phi}{d t^{2}} ; \quad i_{C 1} C_{1} \frac{d u}{d t}$

We have

$i=C_{1} \frac{d u}{d t}+C W \frac{d^{2} \Phi}{d t^{2}}+g W \frac{d \Phi}{d t}+\frac{K}{W} \Phi^{7}$
Fig. 3. Dependences $Z_{m}=f\left(Y_{m}\right)$ for various $\omega$
After the introduction of relative values (8) takes the following form: 
$z=\frac{1}{\beta_{1}} \frac{d y}{d \tau}+\frac{d^{2} x}{d \tau^{2}}+\delta \frac{d x}{d t}+\frac{x^{n}}{A}$

Here

$$
i_{\sigma}=\omega^{2} W C_{2} \Phi_{\sigma} ;\left(\beta_{1}=1 / \omega^{2} L C_{1}\right)
$$

Taking $x=\mathrm{X}_{m} \cdot \sin \tau$, when $\mathrm{y}=\mathrm{y}_{m} \cdot \cos \cdot\left(\tau+\psi_{\mathrm{n}}\right)$, from (9) we obtain

$$
\begin{gathered}
z_{m}^{2}=\left(-\frac{y_{m}}{\beta_{1}} \sin \psi_{n}+\delta X_{m}\right)^{2} \cos \tau+\left(-\frac{y_{m}}{\beta_{1}} \cos \psi_{n}+X^{n}{ }_{m}-X_{m}\right)^{2} \\
\operatorname{tg} \psi_{i}=\frac{-\frac{y_{m}}{\beta_{1}} \sin \psi_{n}+\delta X_{m}}{-\frac{y_{m}}{\beta_{1}} \cos \psi_{n}+X_{m}^{n}-X_{m}}
\end{gathered}
$$

Assuming $\delta=0$ from (10), we have $\psi_{n}=0$

$$
y_{m}=X_{m}\left(X_{m}^{n-1}-1+\beta\right),
$$

Where $\beta=\frac{1}{\omega^{2} L C}$

Calculations and experimental studies have shown that it is convenient to take the value of this coefficient equal to unity.

From (12) we have $y_{m}=X^{n}{ }_{m}$ (13), for this case from (10)

$$
z=\frac{y_{m}}{\beta_{1}}+X_{m}-X_{m}^{7}
$$

Based on dependencies (13) and (14), we construct the functions

$$
X_{m}=f\left(Y_{m}\right) \text { и } Z_{m}=f\left(Y_{m}\right) \quad \text { (fig. 7) for }
$$

various values $\beta_{1}$ for $n=7$.

The effect of current stabilization is clearly noticeable when $\beta_{1}=1.3$. Therefore, when choosing the parameters of the FST scheme, it is necessary to ensure the value of the coefficients for $\beta=1$ and $\beta_{1}=1.3$. Under these conditions, there is no trigger effect and there is a stable falling section on the current - voltage characteristic of a three-element ferroresonant circuit in a wide range of input voltage variations $[1,2,7]$.

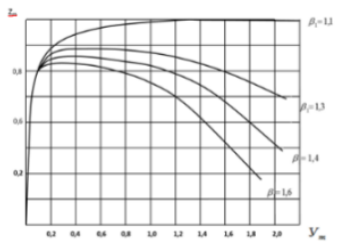

a)

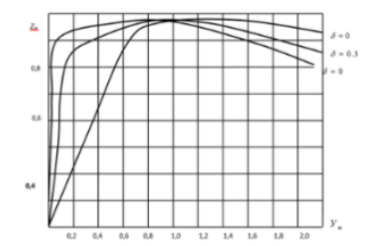

b)
Fig. 7. Dependencies $Z_{m}=f\left(Y_{m}\right)$
Thus, the capacitance $\mathrm{C} 1$ with its linear characteristic provides compensation for the falling part of the characteristic of the three-element oscillatory circuit and significantly affects the nature of the change in the amount of current consumed from the network. The obtained curves (Fig. 7) show that current stabilization, which begins with the appearance of a falling section on the current - voltage characteristic of the EFVCC, can continue even after the resonance point, if the circuit parameters provide parallel extension of the " $\mathrm{S}$ " -shaped characteristic with a linear characteristic of capacitance C1. Therefore, with the right choice of circuit parameters, current stabilization is observed in a very wide range of input voltage changes, which leads to an increase in the output power of the current stabilizer [6].

The proposed FST scheme has a filtering method, which is an important advantage compared to other similar devices. The shape of the stabilized current curve is slightly different from the sine wave, the proportion of higher harmonics is negligible.

\section{References}

1. Filipov E. Nonlinear electrical engineering. -M. Energy 1978-496 s

2. Bessonov L.A. Nonlinear electrical circuits. $-\mathrm{M}$. High school, 1977. -343 p

3. Kadyrov T.M., Rasulov A.N. Single-phase ferroresonant current stabilizer with a three-phase input. University News. Energy 1976, No. 11, pp. 122-124.

4. Kadyrov T.M., Rasulov A.N. Ferroresonant current stabilizer with a sinusoidal current curve. Automation and Telemechanics 1977, No. 11, from 197-200.

5. Milyakh A.N., Volkov I.V. Constant current systems based on inductive capacitive preamplifiers. - Kiev. Naukova Dumka, 1974 - 216 p.

6. M.I. Ibbadullaev, Rasulov A.N. Ruzinazarov M.R. Stabilization of current in two-phase-single-phase circuits. Problems of energy and resource saving No. 3-4. Toshkent 2018.135 - 137 p.

7. Rasulov A.N., Karimov R.CH. Operating mode stabilizer of current on active and inductive loading. European Science Review №9-10. Vina 2015. $140-1431$.

8. I.Rakhmonov Technical science and innovation: Vol.2018 Iss. 1, Article 10. (2018)

9. F.Hoshimov, .Rahmonov, AJTNS, 3-4, 52-55 (2015).

10. F.Khoshimov, I.Rakhmonov, Technical science and innovation: Vol. 2018 : Iss. 1, Article 9. (2018) 